\title{
Changes in the expression of genetic characteristics across cohorts in skeletal deformations of farmed salmonids
}

\author{
Antti KAUSE $^{\mathrm{a} *}$, Ossi Ritola ${ }^{\mathrm{b}}$, Tuija PAANANEN ${ }^{\mathrm{b}}$ \\ ${ }^{a}$ MTT Agrifood Research Finland, Biotechnology and Food Research, Biometrical Genetics, \\ 31600 Jokioinen, Finland \\ ${ }^{\mathrm{b}}$ Finnish Game and Fisheries Research Institute, Tervo Fisheries Research and Aquaculture, \\ 72210 Tervo, Finland
}

(Received 2 November 2006; accepted 24 April 2007)

\begin{abstract}
Genetic analysis of disorder incidence in farmed animals is challenged by two factors. Disorders in different cohorts and environments could be caused by different factors, leading to changes in heritability and to less than unity genetic correlations across cohorts. Moreover, due to computational limitations, liability scale heritabilities at very low incidence may differ from those estimated at higher incidence. We tested whether these two dilemmas occur in skeletal deformations of farmed salmonids using multigeneration data from the Finnish rainbow trout breeding programme and previous salmonid studies. The results showed that heritability was close to zero in cohorts in which management practices maintained incidence at a low level. When there was a management failure and incidence was unusually high, heritability was elevated. This may be due to computational limitations at very low incidence and/or because deformations are induced by different factors in different cohorts. Most genetic correlations between deformations recorded in different generations were weakly to strongly positive. However, also negative correlations between generations were present, showing that high liability at one time can be genetically connected to low liability at another time. The results emphasise that genetic architecture of binary traits can be influenced by trait expression.
\end{abstract}

animal breeding / animal health / deformation / heritability / salmonids

\section{INTRODUCTION}

Improved farmed animal welfare is a fundamental issue for present-day consumers. To ensure profitable and ethical farm animal production and domestication, animal welfare must be addressed. Developmental disorders, such as abnormal development of skeletal structures, occur among both wild and

\footnotetext{
*Corresponding author: Antti.Kause@mtt.fi
} 
farm animals, but the phenomenon is easily exaggerated among farmed animals. This is due to at least three reasons. First, animals with deformations do not survive well under wild conditions [28]. Second, certain deformities arise specially in captivity because of the artificial rearing and management systems $[12,18,21]$. Third, selective breeding for high production may have detrimental effects on health traits [26,31]. Consequently, the occurrence of developmental disorders in farm animals should be controlled by means of improved management practices, and/or by selective breeding when traits have genetic variation.

Animal breeding operates on estimated breeding values, in order to select parents with the highest genetic potential [14,24]. Heritabilities and genetic correlations between traits are needed when estimating breeding values. Modern quantitative genetic studies on farm and wild animals analyse multigenerational data using animal models [14,24]. To obtain estimates of the genetic parameters, a trait recorded on animals originating from multiple generations and multiple environments (e.g., farms) is typically regarded as a single trait for which phenotypic and genetic variances are estimated. This practice has also been adopted for the analysis of disorders and mortality. However, this practice may be unsuitable if deformations and mortality occurring in different cohorts and environments are in fact different traits. This may be revealed by weak positive or even negative genetic correlations, and by different amounts of genetic variation for a trait recorded in different cohorts or environments.

Farmed salmonids are well suited to study whether developmental disorders recorded in different cohorts and environments exhibit different genetic characteristics. Data from health recording systems of fish breeding programmes show that the incidence of deformations changes drastically from place to place and from generation to generation [10, 19,23]. In Finland, skeletal deformations commonly occur at low background incidence $(\leq 2 \%)$. The low incidence is a result of a well-established management system in which all major stressors are under control. Deformations occur at high incidence in rare occasions. This results from a major failure in the management system caused, e.g., by a spread of a disease, unusually warm weather conditions during incubation and early growth phases, or inappropriate feed formulation [3, 21,32,33]. The factors causing deformations do not need to be the same in all cohorts, and deformations induced by different stressors need not share common genetic origin. Thus, there may even be genetic trade-offs (i.e., negative genetic correlations) between resistance mechanisms against alternative stressors [5].

Here we tested whether skeletal deformations in farmed rainbow trout Oncorhynchus mykiss recorded in different production environments and in 
different generations display constant heritability and positive genetic correlations between each other. We tested whether liability scale heritability increases along with increasing incidence for two reasons. First, although heritabilities for binary traits (e.g., $0=$ absent and $1=$ present) calculated on the underlying normally distributed liability scale $[6,8,34]$ should in general be independent of the incidence [29], liability heritability evidently becomes zero when incidence is zero. Thus, it is possible that at very low incidence, liability heritability can be reduced due to computational limitations. Second, it is possible that at an unusually high deformation incidence level, induced by a single major stressor, and families may show consistent differences in response to the major stressor, leading to moderate genetic variation for deformations. In contrast, at background incidence when all major stressors are controlled for, deformations may represent merely developmental noise and/or may be induced by a combination of different factors with very low incidence, both factors leading to low heritability.

We further assessed whether positive or negative genetic correlations exist between deformations recorded in different cohorts and environments. If traits recorded in different cohorts are truly the same trait, genetic correlations between the cohorts tend towards unity. If deformations in different cohorts are induced by different stressors, it is possible that negative genetic correlations appear.

To test these hypotheses, a set of incidences and liability scale heritabilities were obtained from the Finnish rainbow trout breeding programme, and from the previously published salmonid studies [10,23]. Deformation records from discrete generations and environments were treated as separate traits, allowing us to regress the incidences on the heritabilities, and to estimate genetic correlations between the traits.

\section{MATERIAL AND METHODS}

\subsection{Population structure}

The data on the incidence of skeletal deformations were obtained from the Finnish rainbow trout breeding programme. The broodstock fish were held at the fresh water breeding station located in Tervo, Central Finland. The lifecycle of the fish is fully controlled, fish are individually tagged, and their pedigree is maintained over multiple generations [19]. The data included a total of 41286 individuals in five generations hatched in 1993, 1996, 1997, 1999, and 2000 (Tab. I). The pedigree of all fish was known back to generation 1990, in 
Table I. Population structure for each generation.

\begin{tabular}{lccccc}
\hline \multicolumn{5}{c}{ Generation } \\
\hline$n$ sires, $n$ dams & 1993 & 1996 & 1999 & 1997 & 2000 \\
$n$ dams per sire: mean (range) & $5.5(1-9)$ & $2.0(1-4)$ & $2.3(1-4)$ & $2.9(1-5)$ & $2.0(1-5)$ \\
$n$ sires per dam: mean (range) & $2.7(1-3)$ & $1.0(1-1)$ & $1.0(1-1)$ & $2.4(1-3)$ & $1.6(1-3)$ \\
$n$ full-sib families & 247 & 150 & 108 & 191 & 200 \\
$n$ family tanks & 247 & 150 & 116 & 227 & 200 \\
$n$ sea test stations & - & - & 2 & - & 2 \\
\hline
\end{tabular}

$n=$ Sample size

which individuals were assumed to be unrelated. Generation 1990 fish were parents of generation 1993, which was further used to establish generations 1996 and 1997, which share 23 dams and 6 sires born in 1993. Generation 1996 fish were used to produce generation 1999, and generation 1997 fish to produce generation 2000.

In each generation, fish were mated at the fresh water breeding station during April-June using either nested paternal (generations 1996 and 1999) or partial factorial half/full-sib designs (the other generations) (Tab. I). Each time, matings were completed during a one to three week period. In each generation, 47-98 sires and 79-150 dams were mated. Full-sib egg batches were incubated separately, and at the eyed-egg stage, each full-sib family was transported to a separate $150 \mathrm{~L}$ family-tank held indoors. Yet, a total of 68 and 41 full-sib families were allocated into two tanks in generations 1997 and 1999, respectively (Tab. I). Eggs hatched in July.

During the winter after hatching, fingerlings were removed from the family tanks and tagged using Passive Integrated Transponders (Trovan, Ltd., Germany), allowing individual identification throughout the study. An average of 52 randomly chosen fish (range 40-72) from each full-sib family were tagged and held at the fresh water station for grow-out. After tagging, the fish were reared together in the same outdoor raceway for one additional growing season, and recorded for skeletal deformations in April-June. The fish were held in earth-bottom raceways under non-commercial densities (1.5-3.0 individuals $\cdot \mathrm{m}^{-3}$ ).

For generations 1999 and 2000, an additional average of 34 (range 23-86) randomly chosen fish from each full-sib family were further tagged and split into two groups. These groups were transported to two sea stations located in the Baltic Sea (Tab. I). These fish were reared in the same net-pen for one additional growing season, and recorded for deformations from October 
Table II. Incidence, heritability $\left(h^{2}\right)$ and its lower $\left(C L_{\mathrm{low}}\right)$ and higher $\left(C L_{\mathrm{high}}\right) 95 \%$ posterior density interval for seven skeletal deformation traits recorded during five generations and two environments.

\begin{tabular}{lccccccc}
\hline \multicolumn{1}{c}{$\begin{array}{c}\text { Location/ } \\
\text { Generation }\end{array}$} & $\begin{array}{c}\text { Trait } \\
\text { abbreviation }\end{array}$ & $\begin{array}{c}\text { Sample } \\
\text { size }\end{array}$ & $\begin{array}{c}\text { Incidence } \\
(\%)\end{array}$ & $h^{2}$ & $C L_{\text {low }}$ & $C L_{\text {high }}$ \\
\hline $\begin{array}{l}\text { Fresh water station } \\
1993\end{array}$ & G93 & 7910 & 2.00 & 0.06 & 0.027 & 0.129 \\
& & & & & & & \\
1996 & G96 & 5818 & 2.03 & 0.04 & 0.021 & 0.113 \\
1999 & G99 & 3309 & 23.8 & 0.26 & 0.111 & 0.383 \\
& & & & & & \\
1997 & G97 & 8112 & 1.78 & 0.04 & 0.022 & 0.104 \\
2000 & G00 & 6044 & 5.92 & 0.14 & 0.075 & 0.220 \\
& & & & & & & \\
Sea station & G99sea & 2516 & 8.19 & 0.20 & 0.081 & 0.330 \\
1999 & G00sea & 7577 & 4.57 & 0.08 & 0.035 & 0.139 \\
2000 & & & & & &
\end{tabular}

to April. At sea, fish were held in net cages under commercial management conditions.

Recording of all fish in one generation and one rearing location lasted 1 to 2 weeks. At the time of recording, all fish had grown for two growing seasons, and the fish at the fresh and sea water stations weighed an average ( \pm SD) of $1060 \pm 260 \mathrm{~g}$ and $1050 \pm 277 \mathrm{~g}$, respectively.

\subsection{Trait definition}

Deformations were visually recorded based on external characteristics of the live fish. Deformed here refers to fish with deformed skeletal structures of the head, neck, back or tail. A fish with any form of deformation was scored as one and a normal fish as zero. Because the recording is external, the average incidences given are underestimates of the true deformity rates. This is because less destructive deformations (e.g., fusion of two-three vertebrae) may not be identified by the external scoring.

A total of seven separate traits were defined. Each generation, and for generations 1999 and 2000 also, the sea and fresh water environments were treated as separate traits (Tab. II). 


\subsection{Genetic analysis}

We estimated a total of seven heritabilities and incidence levels separately for generations 1993, 1996, 1997, and for the sea and fresh water environments of generations 1999 and 2000 (Tab. II). To estimate heritabilities and genetic correlations on the underlying normally distributed liability scale, we used the MGP-DMU software's animal threshold model applying a Bayesian statistical approach [20]. The animal model used for deformations recorded at the fresh water station was:

$$
y_{\mathrm{i}}=\mu+\operatorname{anim}_{\mathrm{i}}+\varepsilon_{\mathrm{i}}
$$

and for deformations at the sea stations was:

$$
y_{\mathrm{ij}}=\mu+\operatorname{anim}_{\mathrm{i}}+S T A T_{\mathrm{j}}+\varepsilon_{\mathrm{ij}},
$$

where $\mu$ is a population mean, $\operatorname{anim}_{\mathrm{i}}$ is the random genetic animal effect ( $\mathrm{i}=$ number of animals), and $S T A T_{\mathrm{j}}$ is the fixed sea test station effect ( $\mathrm{j}=$ two sea stations), $\varepsilon$ is a random error term, and $y$ is an observation of an individual. We further tested a random full-sib effect without the pedigree information in the model. This effect would have accounted for the variance due to common incubation and initial rearing of full-sib families, and for parts of dominance variance. However, this effect was negligible and thus we excluded it from the models.

The software used Markov chain Monte Carlo (MCMC) methodology to generate marginal posterior distributions of heritabilities and genetic correlations [20]. Heritabilities were obtained from univariate analyses. We ran a chain of 1 million MCMC iterations separately for each trait. From the chain, we saved every 20th estimate, producing a total of 50000 heritability estimates. Genetic correlations were obtained from bivariate analyses. Because MCMC sampling is time consuming, for genetic correlations we reduced the chain length so that we obtained 10000 saved estimates of correlations. The residual correlation between traits was set to zero in each bivariate analysis because a single fish appeared only in one generation and one environment. Flat priors were used because we expected to find differences in heritabilities for the different traits, and potentially both positive and negative genetic correlations between generations [20,27].

From the marginal posterior distributions, we calculated heritability as $h^{2}=$ $V_{\mathrm{G}}\left(V_{\mathrm{G}}+V_{\mathrm{R}}\right)^{-1}$ and genetic correlation as $r_{\mathrm{G}}=C O V_{\mathrm{Gxy}}\left(V_{\mathrm{Gx}} V_{\mathrm{Gy}}\right)^{-0.5}$, where $\mathrm{x}$ and $\mathrm{y}$ are two traits, $V_{\mathrm{G}}$ is genetic variance and $C O V_{\mathrm{G}}$ is genetic covariance. The estimated genetic (co)variances include additive genetic (co)variances but also potential parts of dominance and epistasis (co)variances. The reader 
should note that when calculating a correlation between deformations recorded in different generations and simultaneously in different environments, the effects of generation and environment are confounded.

When heritability reaches zero, or genetic correlation reaches unity or minus one, the posterior distribution becomes skewed. In these cases, we calculated mode rather than mean value, to describe the most common heritability or correlation value. A Bayesian 95\% equal-tailed posterior density interval for genetic parameters was calculated by identifying the cut points leaving $2.5 \%$ of the estimates at the lower and upper tails of the marginal posterior distribution [7].

\section{RESULTS}

\subsection{Relationship between incidence and heritability}

In the Finnish data, the incidence of skeletal deformations ranged from $1.78 \%$ to $23.8 \%$ (Tab. II), the highest values being observed in generations 1999 and 2000. The Finnish data showed that heritabilities were significantly increased when incidence of deformations increased (Fig. 1), as hypothesised. This was evidenced by the regression model (adjusted $R^{2}=95.6 \%, n=7$ ) with statistically significant linear $(3.0 \pm 0.46, t=6.56, p=0.0028)$ and quadratic regression coefficients $(-8.0 \pm 1.76, t=-4.54, p=0.0105)$. The highest heritabilities were observed in generations 1999 and 2000 in fresh and sea water stations. The reader should note that there is a considerable amount of uncertainty in the point estimates, and the $95 \%$ posterior density intervals of different heritabilities are in most cases partly overlapping (Tab. II).

Incidence in the previously published salmonid studies ranged from $2.2 \%$ to $21.5 \%[10,23]$. When the seven Finnish heritability estimates were combined with the five liability scale heritability estimates from these previous studies, the pattern found in the Finnish data remained (Fig. 1). In the regression model of the combined data $\left(R^{2}=79.2 \%, n=12\right)$, both the linear $(5.9 \pm 1.08$, $t=5.44, p=0.0004)$ and quadratic regression coefficients $(-18.9 \pm 4.36$, $t=-4.34, p=0.0019)$ were significant. These results indicate that at low background incidence, heritabilities were close to zero. In contrast, at the incidence of $7.5 \%$ or more, heritabilities ranged from 0.195 to 0.50 .

Within the range of data points available, the heritabilities seemed to reach a plateau at the higher incidence. This may be a true pattern, but the influence of the limited number of heritability estimates as well as the considerable error around the point estimates (e.g., Tab. II) cannot be excluded as an explanation. 


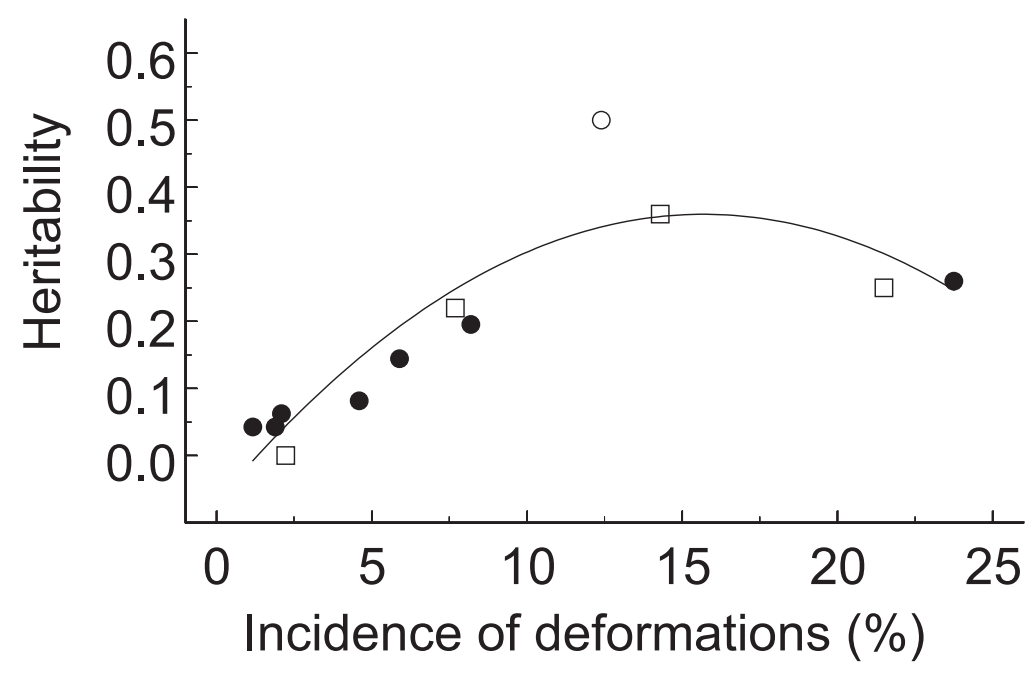

Figure 1. Relationship between incidence and liability scale heritability of skeletal deformations in salmonids. Closed circle (•) indicates an estimate from the Finnish rainbow trout breeding programme $(n=7)$; open circle $(O)$ indicates an estimate for rainbow trout by [23] ( $n=1$ pair of incidence and heritability estimates); open box ( $\square$ ) indicates an estimate for Atlantic salmon by [10] $(n=4)$. The statistically significant quadratic regression line is drawn through all data points.

Thus, we do not feel confident to discuss whether the relationship is linear or non-linear, or what is the detailed shape of the non-linear function.

\subsection{Genetic correlations across generations}

Thirteen out of 19 across-generation correlations of deformation incidence were positive and six were negative (Tab. III). On the one hand, the average of the nine genetic correlations between generations 1993, 1996, 1997 and 1999 was 0.36 (range: 0.01-0.84). For three of these correlations, the 95\% Bayesian equal-tailed posterior density interval did not include zero. On the other hand, the negative genetic correlations were observed only for the pairs of correlations including generation 2000. The average of the ten genetic correlations of generation 2000 with all the other generations was -0.19 (range: $-0.90-0.27$ ), and the $95 \%$ posterior density interval did not include zero for three of the negative correlations (Tab. III). 
Table III. Genetic correlations ${ }^{\mathrm{a}, \mathrm{b}}$ between generations and environments.

\begin{tabular}{lcccccc}
\hline Trait $^{\mathrm{c}}$ & $\mathrm{G} 93$ & $\mathrm{G} 96$ & $\mathrm{G} 97$ & $\mathrm{G} 99$ & G99sea & G00 \\
\hline G93 & $\bullet$ & & & & & \\
G96 & 0.28 & $\bullet$ & & & & \\
G97 & 0.16 & 0.17 & $\bullet$ & & & \\
G99 & 0.34 & 0.01 & 0.18 & $\bullet$ & & \\
G99sea & $0.71^{*}$ & $0.84^{*}$ & $0.59^{*}$ & $0.68^{*}$ & $\bullet$ & \\
G00 & -0.13 & 0.27 & 0.17 & $-0.42^{*}$ & $-0.82^{*}$ & $\bullet$ \\
G00sea & -0.10 & 0.24 & -0.26 & $-0.90^{*}$ & 0.09 & 0.49 \\
\hline
\end{tabular}

a $*=95 \%$ posterior density interval does not include zero.

${ }^{\mathrm{b}}$ All correlations differ significantly from unity.

${ }^{c}$ Traits are defined in Table II.

\subsection{Genetic correlations between environments}

In generation 1999, the genetic correlation between deformations recorded at the fresh water and sea water stations was strongly positive (0.68), and the $95 \%$ posterior density interval did not include zero (0.42-0.85) (Tab. III). For generation 2000, genetic correlation between the environments was positive $(0.49)$ but the $95 \%$ posterior density interval included zero $(-0.01-0.84)$.

\section{DISCUSSION}

\subsection{Changes in heritability}

The first major finding of this study is that improved management conditions decreasing incidence of deformations in salmonids also decreased the amount of genetic variation available for selection. Genetic variation for deformations was low or non-existent at low background incidence, while genetic variation was elevated at unusually high incidence. This allows genetic responses to selection to occur most effectively in extreme environmental conditions when the incidence of deformations is high. When enhancing farm animal performance, improved management practices and selective breeding are typically complementary. However, this was not the case for skeletal deformations in salmonids. Our results show that there is a trade-off between efforts put on management to reduce disorder incidence and genetic potential for selective breeding.

Two not mutually exclusive factors may explain this observation. First, the genetic parameters were here estimated on the underlying liability scale, and thus, the magnitude of genetic variation is expected to be independent of incidence [29]. However, this does not need to apply when incidence is close 
to zero (or unity). The simulation study by van Vleck [29] showed that at an incidence between $5-50 \%$, liability scale heritability of a binary trait appears to be a reasonably unbiased estimate of the true heritability when using sib designs. For a trait with a true heritability of $0.20-0.90$ and incidence of 5-15\%, liability heritabilities were in fact slightly upwards biased, and not downwards biased. However, it is expected that when the incidence approaches zero, liability heritability also decreases. This is because an incidence of $0 \%$ would necessarily lead to a zero heritability estimate, and low incidence complicates the estimation process. To test for this, we generated a series of binary data sets from the normally distributed body weight data obtained from the Finnish breeding programme for rainbow trout. The body weight records were recoded as 0 or 1 at the two sides of the thresholds that produced separate data sets with incidence of $1 \%, 2 \%, 3 \%, 4 \%, 5 \%, 6 \%, 7 \%, 10 \%, 20 \%, 30 \%, 40 \%$ and $50 \%$. Then liability scale heritabilities of the binary traits were calculated following [6] and compared with the heritability of body weight. This practice was repeated for three discrete year classes with heritabilities of $0.26-0.40$ for body weight. The analysis showed that the liability heritability of the binary trait decreased sharply when the incidence decreased from $5 \%$ to $1 \%$ (results not shown). Consequently, the reduction in the liability scale heritability for deformations at low incidence can be caused by an estimation artefact due to the low incidence.

Second, it is possible that different factors caused deformation at different incidence levels, leading to different heritabilities. Some support for this scenario is available. First, some genetic correlations between cohorts were negative. Second, it is possible that when incidence was high, there was a single major stressor inducing the deformations. Under these unexpected conditions, families showed different but consistent sensitivities to the stressor, leading to moderate heritability. In the Finnish data, generations 1999 and 2000 had the highest incidences and the highest heritability for deformations. In both yearclasses, high deformation incidences occurred not only within the breeding programme but also commonly in trout farms located across Finland. Thus, it is likely that one common factor induced the deformations in these generations. In contrast, during the 15 years of running the breeding programme, we were unable to detect any major flaw in the production system that would cause deformations when the incidence is low. Yet, the causes may include a combination of management failures, mechanical damage at egg stage, nutritional deficiency, disease, and random developmental errors, each occurring at very low incidence $[3,21,32]$. Consequently, it is possible that at low background incidence, skeletal deformations are a result of unexplained developmental 
noise with only limited heritable determination. This would prevent us from detecting significant genetic variation for deformations at low incidence. A similar scenario has been suggested for heritability of survival, when it is determined either by a single disease or by multiple diseases or environmental hazards [22].

Heritabilities for leg deformations of chickens [31] and pigs [18], as well as for hip dysplasia of dogs [25] are moderate to high. This indicates that the genetic characteristics of salmonid deformations under eruptive incidence, and not under low incidence, resembles the genetics of developmental disorder in terrestrial farm animals.

Several studies have shown elevated genetic variation for continuously varying traits, such as body size, in stressful or novel environmental conditions, though opposite observations exist especially for wild populations (reviewed by $[4,15,16])$. The most consistent explanation for this pattern is that genetic variation has not been effectively eroded by natural selection in the novel environment $[4,17]$. This may also apply to our observations, but it is difficult to relate these explanations to domestic animals with a short history in captivity.

\subsection{Common genetic determination?}

A common starting point in animal breeding is to assume that a given trait recorded in different cohorts is the same trait, and thus, genetic correlations between the cohorts should be strongly positive. The moderate-to-strong positive genetic correlations found in our data, such as the ones between deformations recorded in generations 1993, 1996, 1997, and 1999, imply that this indeed is the case for deformations. These genetic correlations imply that the deformations in these generations were determined partly by the same set of genes, or a set of closely linked genes.

The second major finding of this study is that genetic correlations between generations may be low or even negative. If deformations at low incidence are a result of developmental noise, then genetic correlations between these generations are expected to be low. Moreover, there were also strong negative genetic correlations between some generations, most consistently between generation 2000 and the other generations. The negative genetic correlations imply that high disorder liability at one time is genetically linked to low liability at the other time. Although speculative, the most likely reason is that different stressors are present during different years, and thus, deformations at different generations do not share common genetic determination. Likewise, work on a fruit fly Drosophila melanogaster has illustrated that effects of different types 
of stress are not always strongly genetically correlated [5]. In fish, genetic correlations between multiple diseases, in turn, have been either positive, negative or approximately zero $[9,11,13]$.

The low positive and negative genetic correlations between generations show that genetic architecture depends on the actual expression of the trait. A trait recorded as skeletal deformation is in fact not a single trait, and its genetic determination varies from place to place and generation to generation. Moreover, because low skeletal deformation incidence is the norm in rainbow trout [19], the very low heritability at low incidence along with the potential negative genetic correlations between some generations hamper selection efforts to reduce deformations.

Genetic correlations between deformations recorded in different environments were significantly or marginally significantly positive. This was in accordance with previous results in Atlantic salmon, where weak family re-ranking occurred across several salt water farms [10]. Similarly, genetic correlations of $0.65-1.00$ have been found for osteoporosis related traits of layer chickens across two production environments [1]. Two mutually nonexclusive explanations exist for our results. First, deformations occurred within each family at an early stage before they were split and distributed to the two production environments. Second, it is possible that after the splitting, the same families were independently subjected to deformations in both environments. On the one hand, the first explanation seems intuitive because egg incubation and early growth are critical phases for skeletal development in fish and other animals $[2,30]$. On the other hand, the second explanation is supported by detailed studies of fish bone structure, showing that deformations may appear in later life stages [33].

Traits such as survival, disease liability, developmental disorders, and many structural traits are typically recorded as binary traits and are present in both farmed and wild species. These often broadly-defined traits are innately determined by multiple underlying factors, but the different factors are not captured in the trait definition when using simple binary classification. In some cases, they also occur at very low incidence. Thus, the phenomenon of spatially and temporally varying genetic variation observed here may also occur for these traits.

In conclusion, the aim of production of farm animals is to be a profitable and ethically accepted enterprise, providing food stuffs for human consumption. Our results highlight that there is an exceptional trade-off between efforts put on management to reduce incidence of deformations and potential for 
selective breeding. We show that for a binary trait such as deformations, the trait expression may change the underlying genetics.

\section{ACKNOWLEDGEMENTS}

We thank Minna Koivula, Ismo Strandén, Stephen Bishop, Cheryl Quinton and two anonymous referees for useful comments on the earlier versions of the text. The study was funded by the Finnish Ministry of Agriculture and Forestry.

\section{REFERENCES}

[1] Bæverfjord G., Åsgard T., Shearer K.D., Development and detection of phosphorus deficiency in Atlantic salmon, Salmo salar L., parr and post-smolts, Aquacult. Nutr. 4 (1998) 1-11.

[2] Bishop S.C., Fleming R.H., McCormack H.A., Flock D.K., Whitehead C.C., Inheritance of bone characteristics affecting osteoporosis in laying hens, $\mathrm{Br}$. Poult. Sci. 41 (2000) 33-40.

[3] Brown C.L., Núñez J.M., Disorders of development, in: Leather J.F., Woo P.T.K. (Eds), Fish Diseases and Disorders, Vol. 2: Non-infectious Disorders, CAB International, Wallingford, 1998, pp. 1-17.

[4] Charmantier A., Garant D., Environmental quality and evolutionary potential: lessons from wild populations, Proc. Royal Soc. London B 272 (2005) $1415-1425$.

[5] Dahlgaard J., Hoffman A.A., Stress resistance and environmental dependency of inbreeding depression in Drosophila melanogaster, Conserv. Biol. 14 (2000) $1187-1192$.

[6] Dempster E.R., Lerner I.M., Heritability of threshold characters, Genetics 35 (1950) 212-236.

[7] Gelman A., Carlin J.B., Stern H.S., Rubin D.B., Bayesian Data Analysis, Chapman \& Hall, London, 1995.

[8] Gianola D., Theory and analysis of threshold characters, J. Anim. Sci. 54 (1982) 1079-1096.

[9] Gjedrem T., Gjøen H.M., Genetic variation in susceptibility of Atlantic salmon, Salmo salar L., to furunculosis, BKD and cold water vibriosis, Aquacult. Res. 26 (1995) 129-134.

[10] Gjerde B., Pante M.J., Bæverfjord G., Genetic variation for a vertebral deformity in Atlantic salmon (Salmo salar), Aquaculture 244 (2005) 77-87.

[11] Gjøen H.M., Refstie T., Ulla O., Gjerde B., Genetic correlations between survival of Atlantic salmon in challenge and field tests, Aquaculture 158 (1997) 277-288.

[12] Gregory N.G., Wilkins L.J., Broken bones in domestic fowl: handling and processing damage in end-of-day battery hens, Br. Poult. Sci. 30 (1989) 555-562. 
[13] Henderson C.R., Best linear unbiased estimation and prediction under a selection model, Biometrics 31 (1975) 423-447.

[14] Henryon M., Berg P., Olesen N.J., Kjær T.E., Slierendrecht W.J., Jokumsen A., Lund I., Selective breeding provides an approach to increase resistance of rainbow trout (Onchorhynchus mykiss) to the diseases, enteric redmouth disease, rainbow trout fry syndrome, and viral haemorrhagic septicaemia, Aquaculture 250 (2005) 621-636.

[15] Hoffman A.A., Merilä J., Heritable variation and evolution under favourable and unfavourable conditions, Trends Evol. Ecol. 14 (1999) 96-101.

[16] Hoffman A.A., Parsons P.A., Evolutionary Genetics and Environmental Stress, Oxford University Press, Oxford, 1991.

[17] Holloway G.J., Povey S.R., Sibly R.M., The effect of new environment on adapted genetic architecture, Heredity 64 (1990) 323-330.

[18] Huang S.Y., Tsou H.L., Kan M.T., Lin W.K., Chi C.S., Genetic study on leg weakness and its relationship with economic traits in central tested boars in subtropical area, Livest. Prod. Sci. 44 (1995) 53-59.

[19] Kause A., Ritola O., Paananen T., Wahlroos H., Mäntysaari E.A., Genetic trends in growth, sexual maturity and skeletal deformations, and rate of inbreeding in a breeding programme for rainbow trout, Aquaculture 247 (2005) 177-187.

[20] Korsgaard I.R., Lund M.S., Sørensen D., Gianola D., Madsen P., Jensen J., Multivariate Bayesian analysis of Gaussian, right censored Gaussian, ordered categorical and binary traits using Gibbs sampling, Genet. Sel. Evol. 35 (2003) 159-183.

[21] Leatherland J.F., Woo P.T.K. (Eds.), Fish Diseases and Disorders. Vol 2: Noninfectious Disorders, CAB International, Wallingford, 1998.

[22] Lush J.L., The Genetics of Populations. Special Report 94, Iowa State University, College of Agriculture, Iowa Agriculture and Home Economics Experiment Station, Ames, Iowa, 1994.

[23] McKay L.R., Gjerde B., Genetic variation for a spinal deformity in Atlantic salmon, Salmo salar, Aquaculture 52 (1986) 263-272.

[24] Mrode R., Linear Models for Prediction of Animal Breeding Values, 2nd edn., CABI publishing, Wallingford, 2005.

[25] Smith G.K., Advances in diagnosing canine hip dysplasia, J. Am. Vet. Med. Assoc. 210 (1997) 1451-1457.

[26] Stern S., Lundeheim N., Johansson K., Andersson K., Osteochondrosis and leg weakness in pigs selected for lean tissue growth rate, Livest. Prod. Sci. 44 (1995) 45-52.

[27] Sørensen D., Gianola D., Likelihood, Bayesian and MCMC Methods in Quantitative Genetics, Springer, New York, 2004.

[28] Tave D., Handwerker T.S., Semi-operculum: a non-heritable birth defect in Tilapia nilotica, J. World Aquacult. Soc. 25 (1994) 333-336.

[29] van Vleck L.D., Estimation of heritability of threshold characters, J. Dairy Sci. 55 (1971) 218-225.

[30] Wagner E.F., Karsenty G., Genetic control of skeletal development, Curr. Opin. Genet. Dev. 11 (2001) 527-532. 
[31] Whitehead C.C., Fleming R.H., Julian R.J., Sørensen P., Skeletal problems associated with selection for increased production, in: Muir W.M. (Ed.), Poultry Genetics, Breeding and Biotechnology, CABI Publishing, Oxon, 2003, pp. 29-52.

[32] Witten P.E., Hansen A., Hall B.K., Features of mono and multinucleated bone resorbing cells of the zebrafish Danio rerio and their contribution to skeletal development, remodeling and growth, J. Morphol. 250 (2001) 197-207.

[33] Witten P.E., Gil-Martens L., Hall B.K., Huysseune A., Obach A., Compressed vertebrae in Atlantic Salmon (Salmo salar): Evidence for metaplastic chondrogenesis as a skeletogenic response late in ontogeny, Dis. Aquat. Org. 64 (2005) 237-246.

[34] Wright S., An analysis of variability in number of digits in an inbred strain of Guinean pigs, Genetics 19 (1934) 506-536. 